\title{
TRPM4 Contributes to Subthreshold Membrane Potential Oscillations in Multiple Mouse Pacemaker Neurons
}

\author{
Keyong Li, Yingtang Shi, Elizabeth C. Gonye, and Douglas A. Bayliss
}

https://doi.org/10.1523/ENEURO.0212-21.2021

Department of Pharmacology, University of Virginia, Charlottesville, VA 22908

\begin{abstract}
Select neuronal populations display steady rhythmic neuronal firing that provides tonic excitation to drive downstream networks and behaviors. In noradrenergic neurons of the locus coeruleus (LC), circadian neurons of the suprachiasmatic nucleus $(\mathrm{SCN})$, and $\mathrm{CO}_{2} / \mathrm{H}^{+}$-activated neurons of the brainstem retrotrapezoid nucleus (RTN), large subthreshold membrane potential oscillations contribute to the pacemaker-like action potential discharge. The oscillations and firing in LC and SCN involve contributions from leak sodium (NALCN) and Ltype calcium channels while recent work from RTN suggested an additional pivotal role for a secondary calcium-activated and voltage-gated cationic current sensitive to TRPM4 channel blockers. Here, we tested whether TRPM4 contributes to subthreshold oscillations in mouse LC and SCN. By RNAscope in situ hybridization, Trpm4 transcripts were detected in both cell groups. In whole-cell recordings from acute slice preparations, prominent voltage-dependent membrane potential oscillations were revealed in LC and SCN after blocking action potentials. These oscillations were inhibited by two chemically-distinct blockers of TRPM4, 9phenanthrol (9-pt) and 4-chloro-2-[[2-(2-chlorophenoxy)acetyl]amino]benzoic acid (CBA). Under whole-cell voltage clamp, inward currents evoked by oscillation voltage waveforms were inhibited in LC by blocking L-type calcium channels and TRPM4. These data implicate TRPM4 in the large subthreshold membrane potential oscillations that underlie tonic action potential discharge in LC and SCN, providing a voltage-dependent and calcium-dependent cationic current to augment the depolarizing inward $\mathrm{Na}^{+}$and $\mathrm{Ca}^{2+}$ currents previously associated with this distinctive electroresponsive property.
\end{abstract}

Key words: calcium-dependent; cationic current; ICaN; tonic firing; TRP channels; waveform command

\section{Significance Statement}

Large subthreshold membrane potential oscillations contribute to spontaneous action potential discharge in select neurons, including those of the locus coeruleus (LC) and the suprachiasmatic nucleus (SCN). The ionic mechanisms underlying these intrinsic membrane properties are incompletely understood. The present study identifies a role for the calcium-activated TRPM4 cation channel in mediating subthreshold oscillations and firing in LC and SCN, supporting previously described roles for NALCN leak sodium channels and L-type calcium channels while generalizing this TRPM4-dependent oscillatory mechanism to multiple pacemaker-type neurons.

Received May 11, 2021; accepted October 21, 2021; First published November 2, 2021.

The authors declare no competing financial interests.
Author contributions: K.L. and D.A.B. designed research; K.L. and E.C.G. performed research; Y.S. contributed unpublished reagents/analytic tools; K.L., E.C.G., and D.A.B. analyzed data; K.L. and D.A.B. wrote the paper. 


\section{Introduction}

The ability to fire action potentials in a regular pacemaker-like pattern is an important intrinsic property of various types of neurons that can, for example, provide tonic excitatory or inhibitory drive to downstream circuits, or a baseline activity level as comparator for dynamic modulation. This regular activity can be sustained in different cell types by a variety of interacting membrane ion channel currents that ultimately determine the firing pattern (Häusser et al., 2004; Bean, 2007). Of particular interest, a large subthreshold membrane potential oscillation has been uncovered that can drive spontaneous action potential discharge. These voltage-dependent oscillations are revealed after blocking fast voltage-activated sodium ( $\mathrm{Nav}$ ) channels, and are typically $\sim 20 \mathrm{mV}$ in amplitude in the 2- to $4-\mathrm{Hz}$ frequency range. Specifically, they have been observed in suprachiasmatic nucleus (SCN) neurons that comprise the circadian clock (Pennartz et al., 2002; Jackson et al., 2004), dopaminergic neurons of the substantia nigra pars compacta (SNpc; Yung et al., 1991; Nedergaard and Greenfield, 1992; Nedergaard et al., 1993; Chan et al., 2007; Puopolo et al., 2007), noradrenergic neurons of the locus coeruleus (LC; Christie et al., 1989; Filosa and Putnam, 2003; Imber and Putnam, 2012; Sanchez-Padilla et al., 2014), and most recently in Nmbexpressing respiratory chemoreceptor neurons in the retrotrapezoid nucleus (RTN; Li et al., 2021).

In terms of ion channel pharmacology, it was recently demonstrated that subthreshold oscillations in RTN neurons depends on both L-type calcium channels (i.e., they were sensitive to dihydropyridine antagonists) and on TRPM4 calcium-dependent cationic channels [i.e., they were sensitive to 9-phenanthrol (9-pt) and 4-chloro-2-[[2(2-chlorophenoxy)acetyl]amino]benzoic acid (CBA); Li et al., 2021]. The seemingly similar oscillations in SCN, SNpc, and LC neurons also include contributions from Ltype calcium channels but a role for TRPM4 channels has not yet been evaluated (Yung et al., 1991; Nedergaard and Greenfield, 1992; Nedergaard et al., 1993; Pennartz et al., 2002; Filosa and Putnam, 2003; Jackson et al., 2004; Chan et al., 2007; Puopolo et al., 2007; SanchezPadilla et al., 2014; Flourakis et al., 2015). Here, we used in situ hybridization, patch clamp recording and ion channel pharmacology to demonstrate that Trpm4 is expressed in LC and SCN neurons, and to show that TRPM4 contributes to subthreshold oscillations observed in those cells in neonatal mouse brainstem slices. The

This work was supported by the National Heart, Lung, and Blood Institute Grant R01 HL108609 (to D.A.B.).

Acknowledgements: We thank Adishesh Narahari for helpful suggestions. We also thank Dr. Hui Chen [University of Virginia (UVA)-Biology] for developing the FFT analysis software and Dr. Pankaj Kumar in the UVA Bioinformatics Core for analysis.

Correspondence should be addressed to Douglas A. Bayliss at bayliss@ virginia.edu.

https://doi.org/10.1523/ENEURO.0212-21.2021

Copyright @ 2021 Li et al.

This is an open-access article distributed under the terms of the Creative Commons Attribution 4.0 International license, which permits unrestricted use, distribution and reproduction in any medium provided that the original work is properly attributed.
TRPM4 current evoked by oscillatory waveform command was particularly sensitive to nifedipine in LC neurons, consistent with a privileged role for L-type channels in supporting the calcium-dependent and voltage-dependent TRPM4 cationic current.

\section{Materials and Methods}

\section{Animals}

Experiments were performed on mice of either sex, following procedures adhering to National Institutes of Health Animal Care and Use Guidelines and approved by the Institutional Animal Care and Use Committee. Mice were housed in Allentown Caging HEPA ventilated racks and steam-sterilized caging (up to four per cage), with crushed corncob bedding and ad libitum access to irradiated Teklad diet (7904 or 7912) and reverse osmosis water provided through an Avidity automatic drinking water system. Animals were exposed to $12 / 12 \mathrm{~h}$ light/dark cycles in a vivarium maintained at $22-24^{\circ} \mathrm{C}$ and $\sim 40 \%$ relative humidity. We used a Phox $2 b:$ :GFP BAC transgenic mouse line (Jx99) that was developed by the GENSAT project and characterized previously (Lazarenko et al., 2009). A total of 67 mice were used (57 neonates for in vitro electrophysiological recording; five adults and five neonates for in situ hybridization experiments).

\section{Mouse brainstem slice preparation}

Brain slices were prepared from neonatal Phox2b::GFP mice at $\sim 4 \mathrm{~h}$ after lights on at 7 A.M. [i.e., at $\sim 11$ A.M., around zeitgeber time (ZT)4; Li et al., 2021]. Briefly, pups [postnatal day (P)6-P14] were anesthetized with ketamine and xylazine (375 and $25 \mathrm{mg} / \mathrm{kg}$, i.m.), rapidly decapitated, and brain slices $(300 \mu \mathrm{m})$ were cut through the LC and SCN regions in ice-cold sucrose-substituted solution, containing the following: $260 \mathrm{~mm}$ sucrose, $3 \mathrm{~mm} \mathrm{KCl,} 5 \mathrm{~mm}$ $\mathrm{MgCl}_{2}, 1 \mathrm{~mm} \mathrm{CaCl}_{2}, 1.25 \mathrm{~mm} \mathrm{NaH}_{2} \mathrm{PO}_{4}, 26 \mathrm{~mm} \mathrm{NaHCO}$, $10 \mathrm{~mm}$ D-glucose, and $1 \mathrm{~mm}$ kynurenic acid. Slices were incubated for $30 \mathrm{~min}$ at $37^{\circ} \mathrm{C}$ and subsequently at room temperature in normal Ringer's solution containing the following: $130 \mathrm{~mm} \mathrm{NaCl}, 3 \mathrm{~mm} \mathrm{KCl}, 2 \mathrm{~mm} \mathrm{MgCl}_{2}, 2 \mathrm{~mm}$ $\mathrm{CaCl}_{2}, 1.25 \mathrm{~mm} \mathrm{NaH}_{2} \mathrm{PO}_{4}, 26 \mathrm{~mm} \mathrm{NaHCO}_{3}$, and $10 \mathrm{~mm} \mathrm{D-}$ glucose. Both cutting and incubation solutions were bubbled with $95 \% \mathrm{O}_{2}$ and $5 \% \mathrm{CO}_{2}$.

\section{In vitro electrophysiology}

Electrophysiological recordings were obtained from ZT5.5 to ZT10 in brain slices from GFP-labeled LC neurons located in the rostral brainstem at the lateral edges of the fourth ventricle or visually identified SCN neurons adjacent to the third ventricle and above the optic chiasm. LC and SCN neurons were recorded at room temperature, a condition that better preserves the health of the slice. We obtained recordings in cell-attached or whole-cell configuration using pClamp, an Axopatch 200B amplifier, and a Digidata 1322A analog-to-digital converter (all from Molecular Devices). For recording, the brainstem slices were placed in a recording chamber mounted on a fluorescence microscope (Zeiss Axioskop FS) in HEPESbased perfusate, containing the following: $140 \mathrm{~mm} \mathrm{NaCl}$, 
$3 \mathrm{~mm} \mathrm{KCl}, 2 \mathrm{~mm} \mathrm{MgCl}_{2}, 2 \mathrm{~mm} \mathrm{CaCl}$, $10 \mathrm{~mm}$ HEPES, and $10 \mathrm{~mm}$ D-glucose. For whole-cell current-clamp recordings or cell-attached voltage-clamp recordings, pipettes were filled with an internal solution containing the following: $17.5 \mathrm{~mm} \mathrm{KCl}, 112.5 \mathrm{~mm}$ K-gluconate, $1.5 \mathrm{~mm} \mathrm{NaCl}, 5$ mm $\mathrm{Na}_{2}$ phosphocreatine, $1 \mathrm{~mm} \mathrm{MgCl} 2,10 \mathrm{~mm}$ HEPES, 0.2 mM EGTA, 3 mm Mg-ATP, and 0.3 mM GTP-Tris (pH 7.2, with $\mathrm{KOH})$. Voltage-clamp recordings of currents evoked by oscillatory waveforms were obtained using a $\mathrm{Cs}^{+}$-based internal solution containing the following: $100 \mathrm{~mm} \mathrm{CsCH} \mathrm{SO}_{3}, 1 \mathrm{~mm}$ $\mathrm{MgCl}_{2}, 0.5 \mathrm{~mm} \mathrm{CaCl}, 5 \mathrm{~mm} \mathrm{Na}$ phosphocreatine, $30 \mathrm{~mm}$ TEACl, $10 \mathrm{~mm}$ HEPES, $10 \mathrm{~mm}$ EGTA, 3 mм Mg-ATP, and 0.3 mm GTP-Tris (pH 7.2, with CsOH). When filled with these solutions, patch electrodes had a DC resistance of 3-6 M $\Omega$; electrode tips were coated with Sylgard 184 (Dow Corning). All reported membrane potentials were corrected for liquid junction potentials measured between the bath and these internal solutions (both $\sim 10 \mathrm{mV}$ ).

In all experiments, a cocktail of blockers was added (10 $\mu \mathrm{M}$ CNQX, $10 \mu \mathrm{m}$ bicuculline, and $30 \mu \mathrm{m}$ strychnine) to inhibit fast excitatory (glutamate) and inhibitory transmitters (GABA, glycine), and subthreshold oscillations were recorded after blocking tetrodotoxin (TTX)-sensitive $\mathrm{Na}_{V}$ channels with TTX $(0.5 \mu \mathrm{M})$. We added $\mathrm{CdCl}_{2}(200 \mu \mathrm{M})$ to the bath to block $\mathrm{Ca}^{2+}$ currents nonspecifically and we applied nifedipine $(10 \mu \mathrm{M})$ to inhibit L-type calcium currents. We used either 9-pt $(30 \mu \mathrm{M})$ or CBA $(50 \mu \mathrm{M})$ as selective TRPM4 channel inhibitors.

After obtaining high-resistance seals $(>1 \mathrm{G} \Omega$ ), cell-attached recordings of spontaneous firing behavior were obtained under voltage clamp at Vhold $=-60 \mathrm{mV}$; the small interspike pipette current at this holding potential (typically less than $\pm 4 \mathrm{pA}$ ) was stable throughout the recordings but may have influenced the baseline spontaneous firing rate measured in LC and SCN neurons (Perkins, 2006). Whole-cell current-clamp recordings of action potentials and subthreshold oscillations were recorded at different membrane potentials achieved by DC current injection. Subthreshold oscillations ( $30 \mathrm{~s}$ ) were analyzed by fast Fourier transform (FFT; MATLAB). The power spectral density (PSD) was computed with the MATLAB function "periodogram" for each frequency bin $(0.03 \mathrm{~Hz})$, and total power calculated from the integrated area of the full power density spectrum. The mean frequency was calculated by MATLAB function "meanfreq." Membrane potential oscillations were captured under current clamp and used as voltage clamp commands to characterize contributions of select currents to the oscillation-waveform trajectory under whole-cell voltage clamp. The blockersensitive currents were obtained by digital subtraction, and the total charge attributed to different currents assessed by integrating the blocker-sensitive currents versus time.

\section{Labeling recorded cells with Lucifer yellow}

Recording pipettes for cell attached recording contained $0.02 \%$ Lucifer yellow. After cell attached recordings were completed, negative pressure was applied to the recording pipette to rupture the membrane. Cells were held in the whole-cell configuration for $3-5 \mathrm{~min}$ to allow exchange of internal solution into the cell, and then a slight amount of positive pressure was applied to the pipette to detach it from the cell. Slices were then placed in 4\% paraformaldehyde (PFA) in phosphate buffer (PB; $0.1 \mathrm{M}$ sodium phosphate) and incubated overnight at $4^{\circ} \mathrm{C}$. After fixation, slices were washed at room temperature in $\mathrm{PB}(3 \times 5$ $\mathrm{min})$, and in Tris saline (TS) buffer $(3 \times 5 \mathrm{~min})$ and blocked for 45 min in 10\% normal horse serum (NHS)/0.3\% Triton X-100/ TS. After blocking, slices were incubated with primary antibody (rabbit anti-Lucifer yellow, 1:1000; Thermo Fisher Scientific A5750, RRID:AB_2536190) in 1\% NHS/0.1\% Triton $\mathrm{X}-100 / \mathrm{TS}$ for $18 \mathrm{~h}$ at $4^{\circ} \mathrm{C}$. Slices were washed in TS $(3 \times 10$ $\mathrm{min}$ ) and then incubated with secondary antibody (donkey anti-rabbit Cy3, 1:500; Jackson ImmunoResearch 711-165152, RRID: AB_2307443) in TS for $90 \mathrm{~min}$ at room temperature. Slices were washed in TS $(3 \times 10 \mathrm{~min})$ and mounted on a charged microscope slide (Fisher 12-550-15) and dried completely before coverslipping with Prolong Gold antifade mounting media containing DAPI (ThermoFisher P36935).

\section{Multiplex in situ hybridization and immunohistochemistry}

Mice were perfused transcardially with $4 \%$ PFA/0.1 M PB. Brains were removed, immersed in the same fixative for $16-18 \mathrm{~h}$ at $4^{\circ} \mathrm{C}$, cut in the transverse plane $(30 \mu \mathrm{m})$ and placed in cryoprotectant (30\% ethylene glycol, $20 \%$ glycerol, and $50 \mathrm{~mm}$ sodium $\mathrm{PB}, \mathrm{pH} 7.4$ ) at $-20^{\circ} \mathrm{C}$ until further processing. Sections were washed in sterile PBS, mounted onto charged slides, and dried overnight. Multiplex in situ hybridization to combine Trpm4 mRNA labeling with detection of either Th, Avp, or Vip transcripts was performed by RNAscope, following manufacturer's instructions [Advanced Cell Diagnostics (ACD): RRID:SCR_012481]. Sections were incubated in hydrogen peroxide $\left(10 \mathrm{~min}, 24^{\circ} \mathrm{C}\right)$, and then in target retrieval solution $\left(5 \mathrm{~min}, 98-102^{\circ} \mathrm{C}\right)$, rinsed in sterile water (x 2), dehydrated in $100 \% \mathrm{EtOH}(5 \mathrm{~min})$, and dried before incubation in protease IV $\left(30 \mathrm{~min}, 40^{\circ} \mathrm{C}\right)$. After rinsing in sterile water $(2 \times)$, sections were incubated with RNAscope catalog oligonucleotide probes for Trpm4, Th, Avp, or Vip (2 $\mathrm{h}, 40^{\circ} \mathrm{C}$ ), and processed using the ACD Multiplex Fluorescent Reagent kit v2. To combine in situ hybridization for Th with immunostaining for GFP, sections were processed for Th labeling as above, using the v1 version ACD Fluorescent Multiplex Detection reagents. Sections were subsequently immunochemically stained for GFP. Briefly, sections were incubated in blocking buffer (10 min; $10 \%$ NHS, 0.1\% Triton X100 diluted in PBS), in primary antibody (1 h; chicken antiGFP, Aves Laboratories, GFP-1020), washed twice with PBS, and then incubated in the secondary antibody $(30 \mathrm{~min}$; donkey anti-chicken Alexa Fluor 488, Jackson ImmunoResearch, 703-545-155). Sections were washed twice with RNAscope wash buffer, air dried and covered with Prolong Gold with DAPI anti-fade mounting medium (Invitrogen).

\section{Statistics}

Results are analyzed and presented using estimation statistics (Bernard, 2019; Calin-Jageman and Cumming, 2019), as described in respective figure legends. Source data for figures are provided in Extended Data 1. 


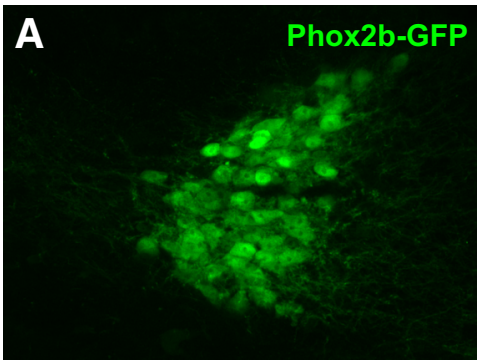

D TTX

$-50 \mathrm{mV}(-11.6 \mathrm{pA})$

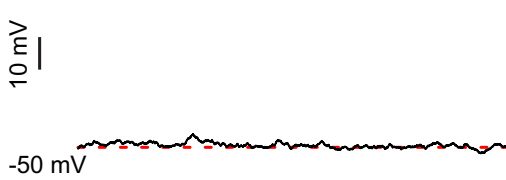

E

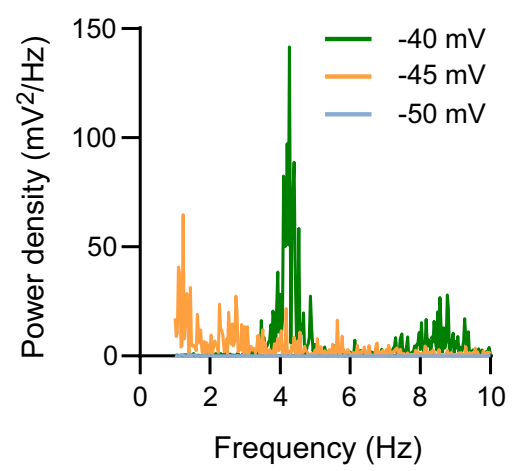

H

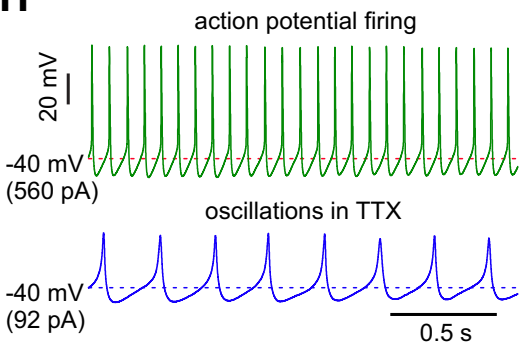

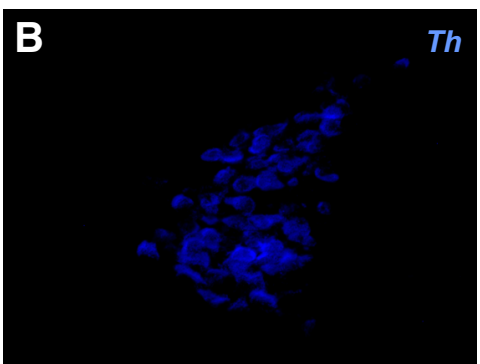

$-45 \mathrm{mV}(26.6 \mathrm{pA})$

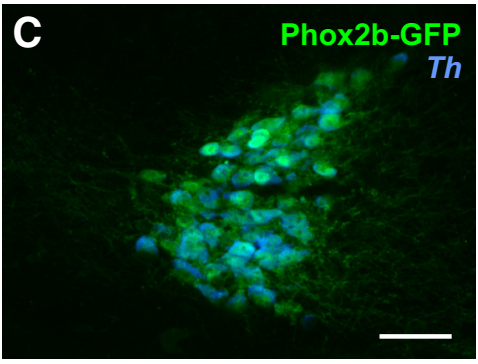

$-40 \mathrm{mV}(92.5 \mathrm{pA})$
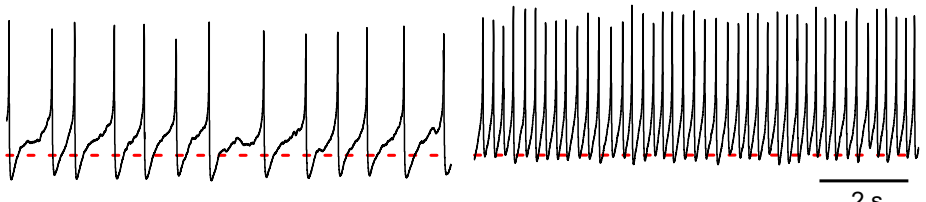

$\mathbf{F}$

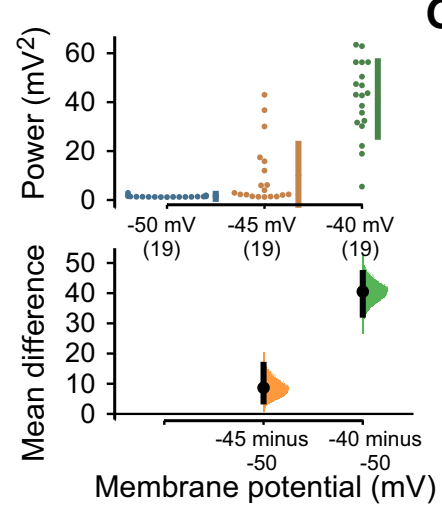

G

I

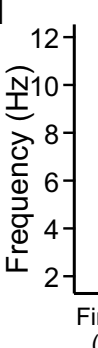

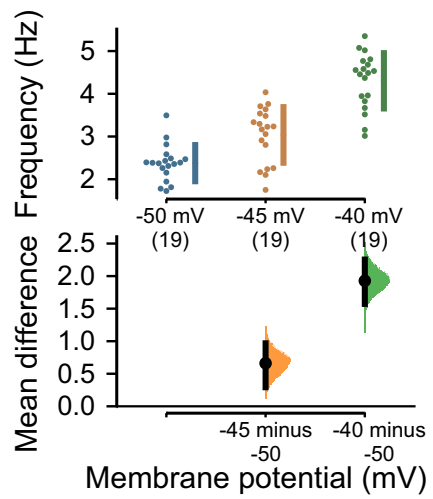

J

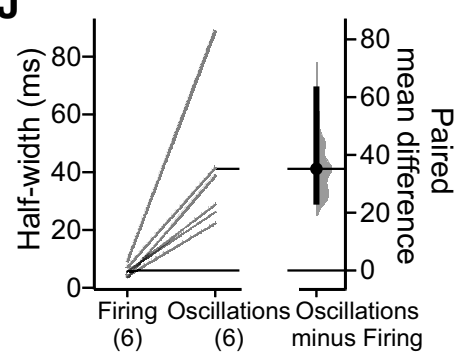

Figure 1. Subthreshold oscillations in LC neurons. A-C, Combined immunohistochemistry for GFP and in situ hybridization for Th in the LC of a Phox2b::GFP mouse. Scale bar: $100 \mu \mathrm{m}$. $\boldsymbol{D}$, Small membrane potential fluctuations at $-50 \mathrm{mV}$ were transformed into large subthreshold oscillations when DC current was injected to depolarize the interoscillation membrane potential. Except where noted, the bath solution in this and all experiments contained TTX $(0.5 \mu \mathrm{M})$ along with fast synaptic blockers $(10 \mu \mathrm{m}$ CNQX, $10 \mu \mathrm{m}$ bicuculline, $30 \mu \mathrm{m}$ strychnine). E-G, Power density-frequency plots for subthreshold oscillations at the indicated membrane potentials were obtained by FFT $(\boldsymbol{E})$, and integrated power $(\boldsymbol{F})$ and mean frequency of subthreshold oscillations $(\boldsymbol{G})$ were plotted at three membrane potentials in the same neurons $(n=19)$. The mean differences from $-50 \mathrm{mV}$ are presented on the lower plots as bootstrap sampling distributions, with mean depicted as a dot and $95 \%$ confidence intervals as vertical error bars. Data were assessed statistically by two-sided permutation $t$ test $(\boldsymbol{F}, p<0.0001$ for -45 and $-40 \mathrm{mV} ; \boldsymbol{G}, p=0.0008$ for $-45 \mathrm{mV}$ and $p<0.0001$ for $-40 \mathrm{mV})$. $\boldsymbol{H}$, Exemplar action potentials and subthreshold oscillations recorded in the same neuron at $-40 \mathrm{mV}$ (by DC current injection). $\boldsymbol{I}$, $\boldsymbol{J}$, Summary data $(n=6)$ depicting the lower frequency $(\boldsymbol{l})$ and longer duration (half-width, $\boldsymbol{J}$ ) of subthreshold oscillations, relative to spontaneous firing. The paired mean differences are presented as bootstrap sampling distributions for firing frequency $(I, p=0.03)$ and for half-width $(\boldsymbol{J}, p<0.0001)$, analyzed by two-sided permutation $t$ test.

\section{Results}

\section{A prominent subthreshold oscillation in LC neurons}

In Phox2b::GFP mice, the noradrenergic LC can be identified by its location in the rostral brainstem at the lateral edges of the fourth ventricle (from $\sim 5.34$ to $5.68 \mathrm{~mm}$ caudal to bregma; Paxinos and Franklin, 2001), and by neuronal co-expression of tyrosine hydroxylase $(T h)$ and GFP (Fig. $1 A-C$ ). In brainstem slice preparations from Phox2b::GFP mice, whole-cell current-clamp recordings in GFP-expressing LC neurons revealed a characteristic tonic action potential discharge under conditions where fast synaptic input is blocked (Li and Putnam, 2013). After eliminating action potential firing with $\Pi \mathrm{TX}(0.5 \mu \mathrm{M})$, a large 
voltage-dependent subthreshold oscillation was revealed when DC current injection was used to progressively depolarize the membrane potential beyond $-50 \mathrm{mV}$ (Fig. 1D). The oscillations were analyzed by FFT in individual LC neurons at membrane potentials from -50 to $-40 \mathrm{mV}$ $(n=19)$, with the strongest frequency components appearing in the 2- to $4-\mathrm{Hz}$ band across that voltage range (Fig. 1E). The subthreshold oscillations increased in power and frequency at more depolarized potentials (Fig. $1 F, G)$, and consistently occurred at lower frequencies and with longer durations than the corresponding action potentials recorded in the same cells before TTX (Fig. 1H-J). The properties of these depolarization-evoked subthreshold oscillations are similar to those described previously in LC neurons, where they were variously called TTX-insensitive spikes (Filosa and Putnam, 2003; Imber and Putnam, 2012) or "spikelets" (Sanchez-Padilla et al., 2014).

\section{TRPM4 channels underlie subthreshold oscillations in LC neurons}

A similar subthreshold oscillation in RTN chemosensory neurons requires activity of L-type calcium channels and, secondarily, the calcium-activated TRPM4 nonspecific cation channel (Li et al., 2021). In previous work from LC neurons, the subthreshold oscillation (i.e., TTX-insensitive spikes and spikelets) was also attributed to L-type calcium channels but involvement of TRPM4 was not assessed (Filosa and Putnam, 2003; Imber and Putnam, 2012; Sanchez-Padilla et al., 2014).

Expression of Trpm4 (and Trpm5 to a lesser extent) has been identified by qRT-PCR from microdissections of the LC region (Cui et al., 2011), and in a microarray analysis, Trpm4 was found to be 17-fold enriched in LC neurons, relative to hindbrain (Mulvey et al., 2018). Therefore, we first performed multiplex fluorescence in situ hybridization to examine Trpm4 expression specifically in catecholaminergic neurons of the LC, identified by co-expression of Th. We found Trpm4 transcript labeling in Th-expressing LC neurons in sections from both neonatal and adult mice (Fig. 2A) at levels apparently similar to (adult) or slightly less (neonate) than observed in the mesencephalic trigeminal (MeV) cells located just laterally (Fig. 2A, arrows). Therefore, we used two chemically distinct TRPM4 channel blockers, 9-pt $(30 \mu \mathrm{M})$ and CBA $(50 \mu \mathrm{m})$, to test the contributions of TRPM4 to TTX-resistant subthreshold oscillations and their underlying currents in LC neurons (Grand et al., 2008; Guinamard et al., 2014; Burris et al., 2015; Ozhathil et al., 2018). At these concentrations, and consistent with previous results from RTN neurons ( $\mathrm{Li}$ et al., 2021), we observed a strong reduction in both the power and frequency of the oscillations in LC neurons after exposure to either of the TRPM4 blockers (Fig. 2B$G)$. In both cases, a residual oscillation remained, perhaps reflecting incomplete TRPM4 block at the applied concentrations or contributions from other channels.

\section{Oscillations evoke calcium and TRPM4 currents in LC neurons}

To examine contributions of calcium and TRPM4 channels activated during the membrane potential oscillation, we used whole-cell voltage clamp to measure ionic currents evoked by an oscillation waveform command derived from LC neurons (Fig. $3 A$, upper). Oscillationwaveform-evoked currents were recorded in the presence of TTX and fast synaptic blockers, and using a $\mathrm{Cs}^{+}$based, TEA-containing pipette solution to eliminate $\mathrm{K}^{+}$ currents. The oscillation waveform elicited a biphasic inward current under control conditions, with an initial inward peak just before the waveform reached the maximal depolarization followed by a second, and larger, inward current peak during the repolarizing phase of the waveform (Fig. $3 A$, lower, dashed blue line). These oscillation waveform-evoked currents include passive leak and capacitive components, together with contributions from the ion channel currents of interest; the latter were isolated as the components of waveform-evoked current that are sensitive to various ion channel blockers.

We first exposed cells to 9-pt to test whether TRPM4 currents were activated during the oscillation voltage waveform. The 9-pt-sensitive current was revealed by digital subtraction of currents measured in the presence of 9-pt from those obtained under control conditions (Fig. $3 A$, lower, orange line). This 9-pt-sensitive, TRPM4-like current was also biphasic, with an initial inward current peak preceding the maximum depolarization and a second peak during the repolarizing phase of the waveform. When transformed against voltage, the 9-pt-sensitive current was inward throughout the oscillation waveform, with the two inward current peaks evident as a loop moving toward and away from the peak voltage in the current-voltage (I-V) plot (Fig. 3B, orange).

TRPM4 is a calcium-activated cationic channel, and previous observations in LC neurons have suggested a prominent role for voltage-activated calcium currents in generating the oscillations (Filosa and Putnam, 2003; Imber and Putnam, 2012; Sanchez-Padilla et al., 2014). Thus, to characterize the calcium current components evoked by the oscillation voltage waveform, we next tested effects of the nonspecific calcium channel block$\mathrm{er}, \mathrm{Cd}^{2+}(200 \mu \mathrm{M}$, in the continued presence of 9-pt). The $\mathrm{Cd}^{2+}$-sensitive current was much larger in amplitude than the 9-pt-sensitive current, although similarly biphasic, with two distinct peaks coinciding in both voltage and time with those of the 9-pt-sensitive current (Fig. $3 A-C$, green); these properties were also reflected in the $\mathrm{I}-\mathrm{V}$ plot for the $\mathrm{Cd}^{2+}$-sensitive current, that was again inward throughout the waveform (Fig. 3B). The two peaks of $\mathrm{Cd}^{2+}$-sensitive current presumably result from activation/inactivation properties of different calcium channels with distinct voltage-dependence and kinetics, as those play out over the continually changing driving force for $\mathrm{Ca}^{2+}$ throughout the voltage waveform (i.e., decreasing during the depolarizing phase and then increasing again during membrane repolarization). The biphasic nature of the $\mathrm{Ca}^{2+}$-dependent TRPM4 cationic current (i.e., 9-ptsensitive current) likely reflects similar changes in sodium driving force throughout the waveform, while also being influenced by the kinetics of the $\mathrm{Ca}^{2+}$ current evoked by the waveform. Consistent with the expected 

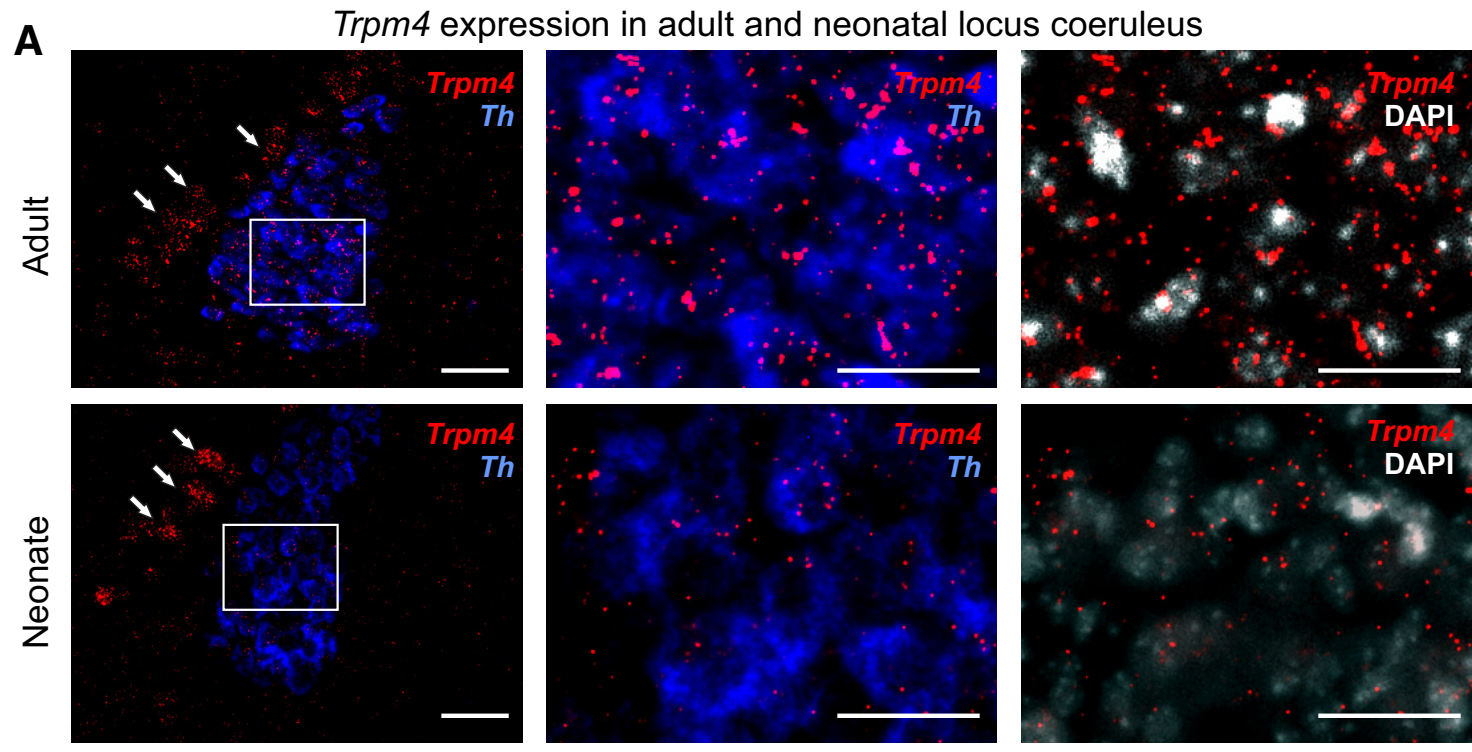

B

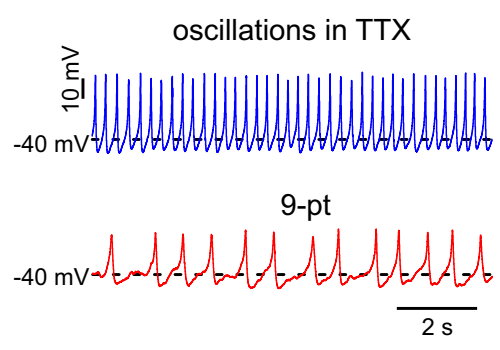

E
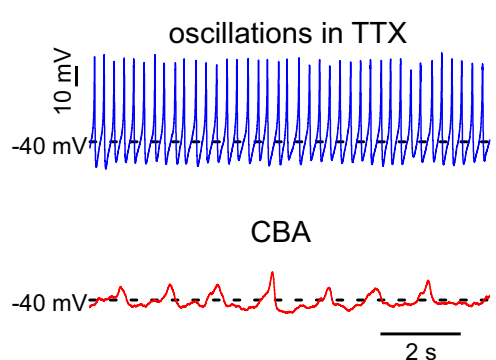

C

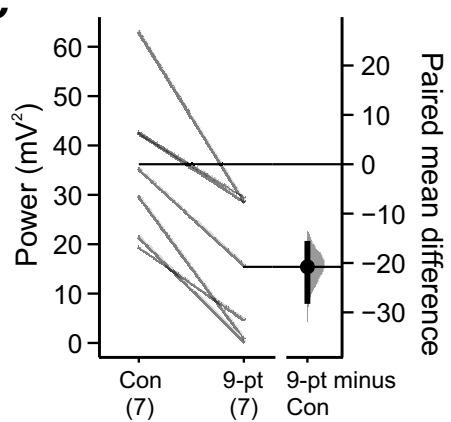

$\mathbf{F}$

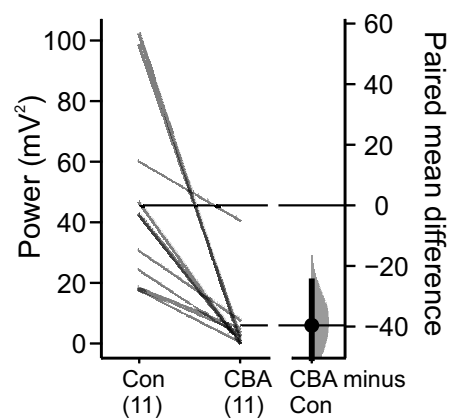

D

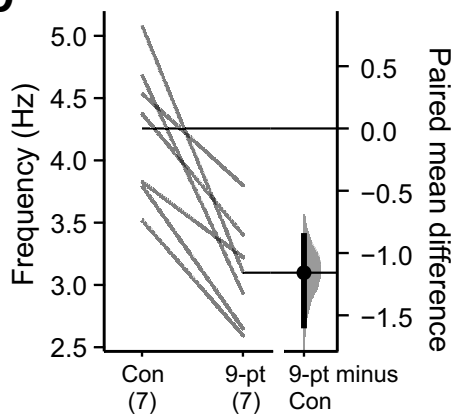

G

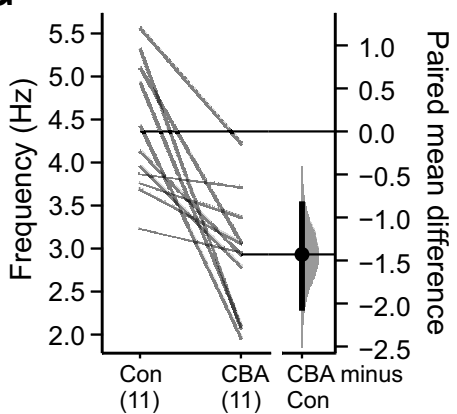

Figure 2. TRPM4 is expressed and contributes to subthreshold oscillations in LC neurons. $\boldsymbol{A}$, RNAscope analysis of Th (blue) and Trpm4 (red) transcript expression in mouse LC neurons from adult (upper) and neonate (lower). The boxed regions in lower power images are shown at higher power (scale bars: $50 \mu \mathrm{m}$ ). Arrows point to laterally located MeV neurons also expressing Trpm4. B-G, Exemplar effects on subthreshold oscillations in TTX $(0.5 \mu \mathrm{M}$ TTX, at $-40 \mathrm{mV}$ with DC current injection) of bath application of two TRPM4 channel blockers, 9-pt $(\boldsymbol{B}, 30 \mu \mathrm{M})$ and CBA $(\boldsymbol{E}, 50 \mu \mathrm{M})$, with summary for all cells showing paired mean differences as bootstrap sampling distributions for effects on integrated power $(\boldsymbol{C}, \boldsymbol{F})$ and mean frequency $(\boldsymbol{D}, \boldsymbol{G})$ after either 9 -pt $(\boldsymbol{C}, \boldsymbol{D})$ or $\mathrm{CBA}(\boldsymbol{F}, \boldsymbol{G})$; $p<0.0001$ for all paired mean differences by two-sided permutation $t$ test.

$\mathrm{Ca}^{2+}$-dependence of TRPM4, when $\mathrm{Cd}^{2+}$ was applied to block $\mathrm{Ca}^{2+}$ current before 9-pt exposure, the oscillation no longer evoked any 9-pt-sensitive current (Fig. 3D). This analysis indicates that $\mathrm{Ca}^{2+}$ channels and $\mathrm{Ca}^{2+}$-dependent TRPM4 channels contribute to the membrane potential oscillation in LC neurons, with $\mathrm{Ca}^{2+}$ channels accounting for the predominant current component.
In previous work from LC neurons, the subthreshold membrane potential oscillations were strongly inhibited by dihydropyridine L-type calcium channel antagonists (Filosa and Putnam, 2003; Imber and Putnam, 2012; SanchezPadilla et al., 2014). Therefore, we tested whether a nifedipine-sensitive, L-type calcium current was evoked by the oscillation waveform in LC neurons. The nifedipine-sensitive 

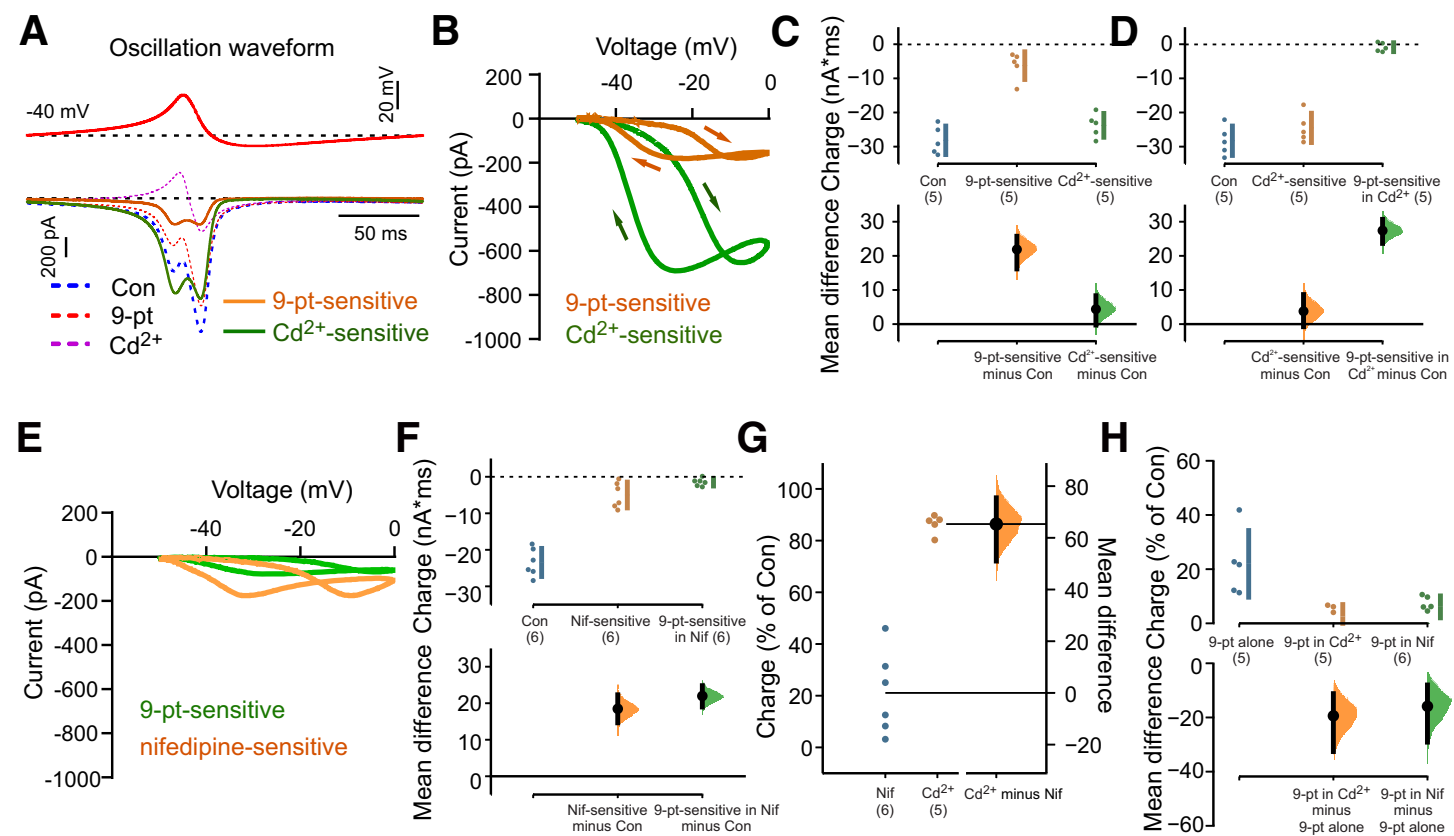

Figure 3. Calcium channels (L-type) and calcium-dependent TRPM4 currents contribute to subthreshold oscillations in LC neurons. $\boldsymbol{A}$, upper, Oscillation-waveform voltage command applied from Vhold $=-40 \mathrm{mV}$ in LC neurons recorded with a Cs ${ }^{+}$-based pipette solution. Lower, Oscillation-waveform-evoked currents under control conditions, in the presence of 9-pt (30 $\mu \mathrm{M})$ and in the presence of $200 \mu \mathrm{M} \mathrm{Cd}^{2+}$ (dashed lines); 9-pt-sensitive and $\mathrm{Cd}^{2+}$-sensitive current components (thick lines) were obtained by digital subtraction. $\boldsymbol{B}$, I-V curves of oscillation-waveform-induced currents reveal inward 9-pt-sensitive and $\mathrm{Cd}^{2+}$-sensitive currents throughout the oscillation-waveform; in this experiment, 9-pt was applied before $\mathrm{Cd}^{2+} . \boldsymbol{C}, \boldsymbol{D}$, Summary data show the relative contributions of 9-pt-sensitive and Cd ${ }^{2+}$-sensitive currents during the oscillation waveform command $(\boldsymbol{C})$ and illustrate that the 9-pt-sensitive current was eliminated when calcium currents were previously blocked with $\mathrm{Cd}^{2+}(\boldsymbol{D})$. The mean differences from control are presented on the lower plots as bootstrap sampling distributions. Data were assessed statistically by two-sided permutation $t$ test $\left(\boldsymbol{C}, p=0.0012\right.$ for 9 -pt-sensitive and $p=0.114$ for $\mathbf{C d}^{2+}$-sensitive; $\boldsymbol{D}, p=0.195$ for $\mathrm{Cd}^{2+}$-sensitive and $p=0.0012$ for 9 -pt-sensitive in $\left.\mathrm{Cd}^{2+}\right)$. E, Exemplar I-V curves of oscillation-waveform-induced currents during exposure to the L-type channel blocker nifedipine $(10 \mu \mathrm{M})$ and then 9-pt $(30 \mu \mathrm{M})$. $\boldsymbol{F}$, Summary data $(n=6)$ showing the relative contributions of nifedipine-sensitive and 9-pt-sensitive currents (after nifedipine) during the oscillation waveform command. The mean differences from control are presented on the lower plots as bootstrap sampling distributions. Data were assessed statistically by two-sided permutation $t$ test $(p<0.0001$ for nifedipine and for 9-pt in nifedipine). G, Summary of the percentage of total charge during the waveform attributed to calcium current $\left(\mathrm{Cd}^{2+}\right.$-sensitive) and L-type (nifedipine-sensitive) calcium channel current. The mean differences from Cd ${ }^{2+}$ are presented on the lower plots as bootstrap sampling distributions. For $\mathrm{Cd}^{2+}$-sensitive $(n=5)$ versus nifedipine-sensitive $(n=6): p<0.0001$, by two-sided permutation $t$ test. $\boldsymbol{H}$, Summary of the percentage of total charge attributed to TRPM4 current under the indicated conditions. The mean differences from 9-pt-sensitive $(n=5)$ : $p=0.006$ for 9-pt-sensitive in $\mathrm{Cd}^{2+}(n=5)$, and $p=0.003$ for 9-pt-sensitive in nifedipine $(n=6)$ by twosided permutation $t$ test.

waveform-evoked current displayed I-V characteristics similar to the total waveform-evoked $\mathrm{Ca}^{2+}$ current (Fig. 3E,F), but it was much smaller in amplitude than the overall $\mathrm{Cd}^{2+}$-sensitive current (compare with Fig. 3D). Likewise, the 9-pt-sensitive current was still present but reduced in amplitude after blocking L-type channels with nifedipine (Fig. 3E,F). Overall, $\mathrm{Cd}^{2+}$-sensitive current carried the largest fraction of charge during the oscillation waveform $(\sim 80 \%)$, including $\sim 20 \%$ that was dependent on L-type calcium channels (Fig. 3G). Approximately $20 \%$ of the inward charge could be ascribed to 9-pt-sensitive, TRPM4 channel current and, interestingly, although L-type channels accounted for only $\sim 25 \%$ of the total charge because of $\mathrm{Ca}^{2+}$ current, blocking those channels with nifedipine reduced the 9-pt-sensitive current by nearly as much as $\mathrm{Cd}^{2+}$ (i.e., by $\sim 70 \%$; Fig. $3 H$ ). This suggests a preferential, although not exclusive, coupling between L-type $\mathrm{Ca}^{2+}$ channels and $\mathrm{Ca}^{2+}$-dependent TRPM4 channels.

\section{TRPM4 regulates spontaneous cell firing in LC neurons}

To test whether TRPM4 plays a role in spontaneous pacemaker-like activity in LC neurons, we determined effects of 9pt on action potential discharge using cell-attached recordings; this patch clamp configuration avoids disrupting the internal milieu of the recorded neuron. As illustrated for the exemplar LC neuron in Figure $4 A$, the steady metronomic-like firing typically observed under control conditions was reduced in frequency after bath application of 9-pt (30 uM). This 9-pt-induced decrease in firing frequency was consistently noted across all cells tested (Fig. 4B), indicating that TRPM4 participates in determining $L C$ firing frequency. As we observed for its effects on subthreshold oscillations (Fig. $2 B-D$ ), spontaneous firing was not eliminated by 9 -pt at this concentration.

\section{TRPM4 is expressed at low levels in SCN neurons}

Subthreshold voltage-dependent membrane potential oscillations have been recorded in circadian-associated 
A

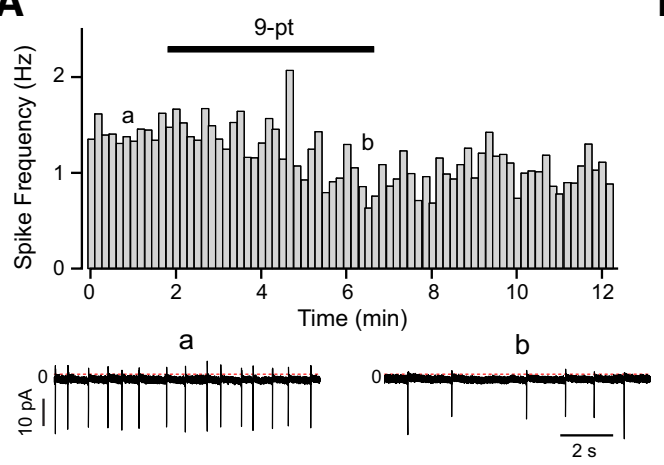

B

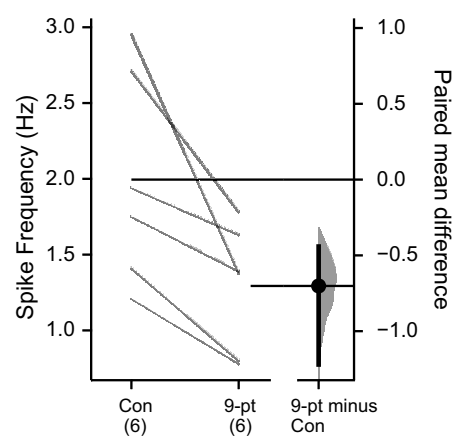

Figure 4. TRPM4 contributes to spontaneous firing in LC neurons. $\boldsymbol{A}$, Rate histogram (10-s bin averages of instantaneous frequency) of spontaneous action potential firing (no TTX) in an LC neuron recorded in cell-attached configuration, under control conditions and in the presence of 9-pt (30 $\mu \mathrm{m})$. Example recordings from the indicated time points are provided (below). $\boldsymbol{B}$, Summary data show firing frequency for cells in control and 9-pt, and the paired mean difference between control and 9-pt as a bootstrap sampling distribution; $p<0.0001$ by two-sided permutation $t$ test.

SCN neurons during the daytime, a period when SCN neurons are depolarized and fire spontaneously at higher frequencies than during the night (Pennartz et al., 2002; Jackson et al., 2004). A complex set of channels has been implicated in mediating the oscillations and cell firing, but a role for TRPM4 has not been tested (Pennartz et al., 2002; Jackson et al., 2004; Flourakis et al., 2015).

We first used multiplex RNAscope to assess Trpm4 transcript expression in SCN neurons from adult and neonatal mice. The SCN is a heterogenous nucleus with at least five molecularly defined neuronal cell groups, each of which shows some degree of circadian rhythmicity (Wen et al., 2020). Among these, three distinct subsets of SCN neurons express either (or both) of the neuropeptides, vasoactive intestinal polypeptide (Vip) or arginine vasopressin (Avp), which are associated, respectively, with the core and shell regions of the SCN (Card et al., 1981; Abrahamson and Moore, 2001; Todd et al., 2020; Wen et al., 2020). Thus, to aid in SCN localization for these anatomic studies, we combined Trpm4 localization with labeling for Vip and Avp. A low level of Trpm4 expression was detectable in the SCN of both adult (Fig. 5A, upper) and neonate mice (Fig. $5 A$, lower) that was not obviously restricted to either the $\mathrm{Vip}^{+}$and $\mathrm{Avp}^{+}$neurons. As a reference control, note that the relative levels of Trpm4 expression in SCN were roughly comparable to those in CA1 pyramidal cell region (Fig. 5Bi), and higher than the apparently background levels in stratum radiatum (Fig. $5 B$ ii). These data suggest that TRPM4 channels are expressed in SCN neurons where they could contribute to subthreshold oscillations.

\section{TRPM4 in SCN neurons contributes to subthreshold oscillations}

We next tested whether TRPM4 channels contribute to the subthreshold voltage-dependent membrane potential oscillations observed previously in SCN neurons (Pennartz et al., 2002; Jackson et al., 2004). We prepared brain slices from the mouse ventral forebrain and recorded from visually-identified neurons in SCN, recognizable as a cell-dense region at the base of the third ventricle immediately superior to the optic chiasm (from 0.82 to $0.34 \mathrm{~mm}$ caudal to bregma; Paxinos and Franklin, 2001). Recordings were not limited to the dorsomedial (shell) regions of the SCN, where oscillations were previously observed (Pennartz et al., 2002; Jackson et al., 2004).

In the presence of synaptic blockers, SCN neurons fired spontaneous action potentials that were blocked with TTX, often revealing a slower, large amplitude subthreshold oscillation (Fig. 6A; Pennartz et al., 2002; Jackson et al., 2004). In the presence of TTX, these subthreshold membrane potential oscillations were uncovered or enhanced in a substantial fraction of cells as they were depolarized with DC current injection (Fig. 6B). The membrane potential oscillations in SCN neurons were analyzed by FFT; in those neurons where they were evident $(n=24 / 31, \sim 77 \%)$, the subthreshold oscillations were voltage-dependent, increasing in power and frequency at depolarized membrane potentials up to $\sim-45 \mathrm{mV}$, with no further increase at $-40 \mathrm{mV}$ (Fig. 6C,D). We tested whether TRPM4 contributes to these oscillations in SCN neurons using 9-pt and CBA. Indeed, both TRPM4 blockers nearly eliminated the oscillations (Fig. $6 E, H)$; interestingly, although the oscillation power was reduced by the blockers (Fig. 6F,I), the remaining membrane potential fluctuations continued at the same frequency as the larger oscillations (Fig. 6G,J).

\section{TRPM4 influences spontaneous cell firing in SCN neurons}

Finally, we examined whether TRPM4 contributes to spontaneous action potential firing in SCN neurons. As depicted in Figure $7 A$, by cell-attached recording, we found that 9-pt decreased firing rate in SCN neurons; after whole-cell access with a Lucifer yellow-filled pipette and post hoc staining, we confirmed the cell locations in the SCN (Fig. 7B). This response was typical of all SCN neurons tested, in which the TRPM4 blocker consistently reduced baseline firing frequency without eliminating spontaneous firing (Fig. 7C). Thus, TRPM4 contributes to setting basal discharge rate in SCN neurons. 
A

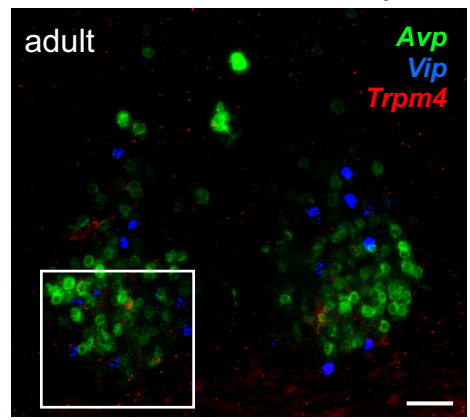

Trpm4 expression in adult and neonatal SCN
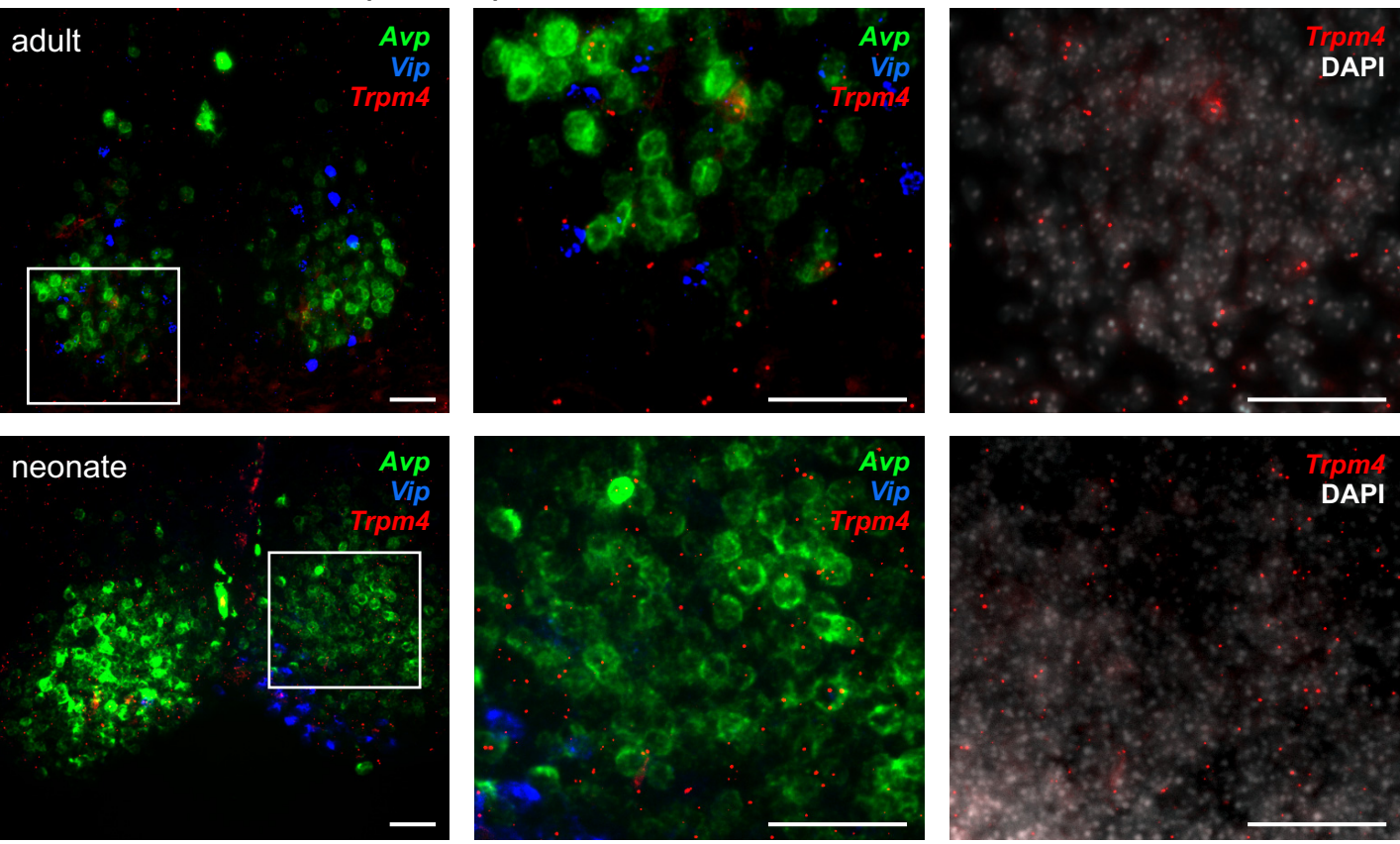

B

Trpm4 expression in neonatal CA1 hippocampus
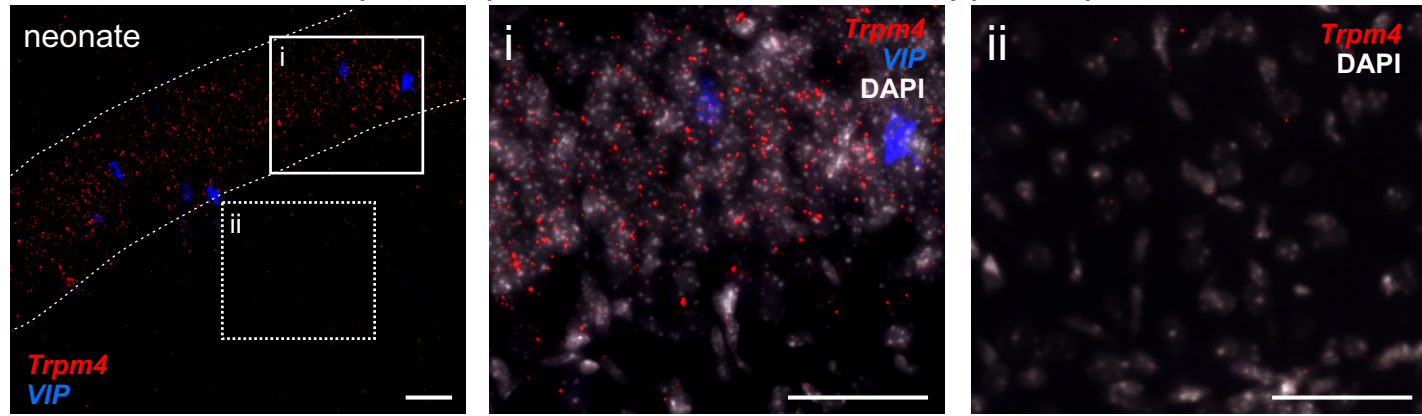

Figure 5. Trpm4 expression in SCN adult and neonatal SCN neurons. A, RNAscope analysis of Avp (green), Vip (blue), and Trpm4 (red) transcript expression in mouse SCN neurons from adult (upper) and neonate (lower). The boxed regions in lower power images are shown at higher power (and with a DAPI counterstain, right). B, RNAscope analysis of Vip (blue) and Trpm4 (red) in neonatal mouse hippocampus, with magnified images from the boxed regions shown with a DAPI counterstain to illustrate moderate Trpm4 transcript levels (Bi; CA1) and background levels of labeling (Bii; SR, stratum radiatum). Dashed lines outline the CA1 region in $\boldsymbol{B}$; scale bars: $50 \mu \mathrm{m}$.

\section{Discussion}

Subthreshold membrane potential oscillations are an uncommon, but important electroresponsive property that support spontaneous pacemaker-like action potential discharge in select neuronal populations. In earlier work, voltage-dependent TTX-resistant subthreshold oscillations were observed in LC and SCN neurons and, based on their sensitivity to dihydropyridine antagonists, L-type calcium channels were associated with those oscillatory events (Pennartz et al., 2002; Filosa and Putnam, 2003; Jackson et al., 2004; Imber and Putnam, 2012; SanchezPadilla et al., 2014). The current work extends that idea by demonstrating the additional involvement of TRPM4, a calcium-activated and voltage-dependent cationic channel in the oscillations of both LC and SCN neurons. This recalls the recent description of an overtly similar subthreshold membrane potential oscillation in mouse RTN neurons that was also dependent on L-type calcium channels and TRPM4 (Li et al., 2021). Thus, it appears that subthreshold oscillations require calcium entry, along with a depolarizing current, to activate TRPM4 channels and drive the oscillations in RTN, LC, and SCN neurons. In these neurons, we found that pharmacological inhibition of TRPM4 reduced, but did not eliminate, baseline action potential discharge rate (this work, and Li et al., 2021). Thus, although TRPM4 activity is necessary for the large amplitude TTX-resistant membrane potential oscillations and modulates firing rate in these neurons, it is not absolutely required for spontaneous pacemaker-like firing.

\section{TRPM4 and other channels regulate neuronal oscillations and firing}

The subthreshold membrane potential properties that underlie spontaneous firing activity in LC and SCN neurons 
A

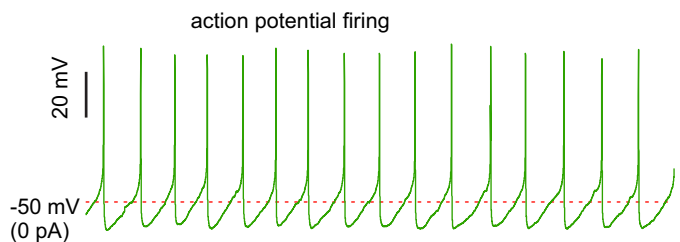

oscillations in TTX

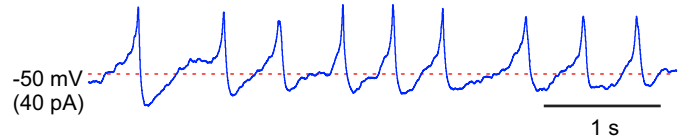

B

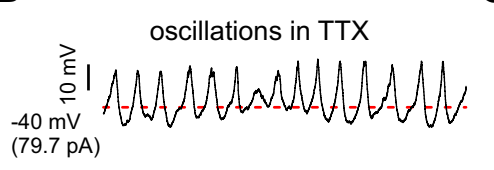

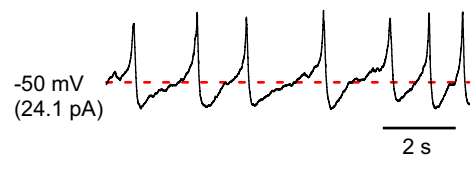

E

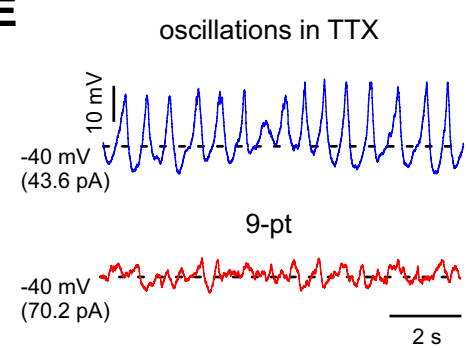

H

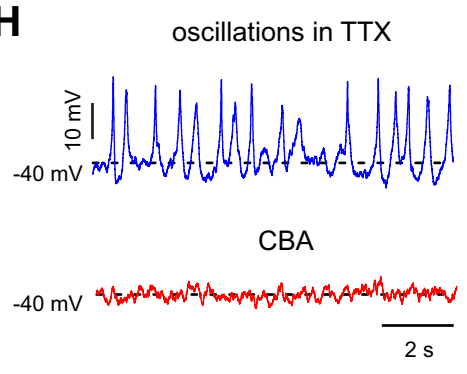

C
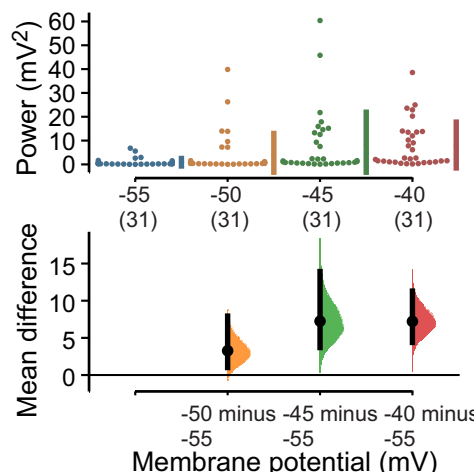
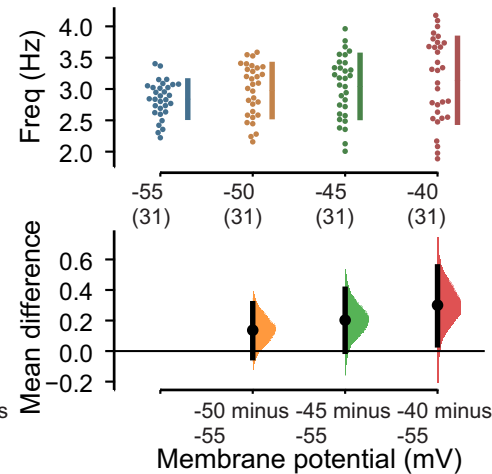

F

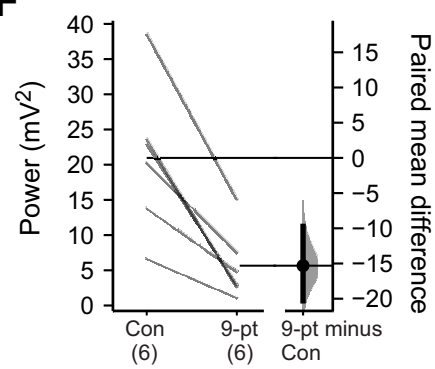

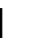

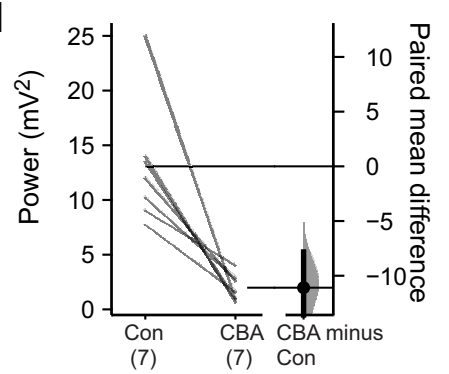

G

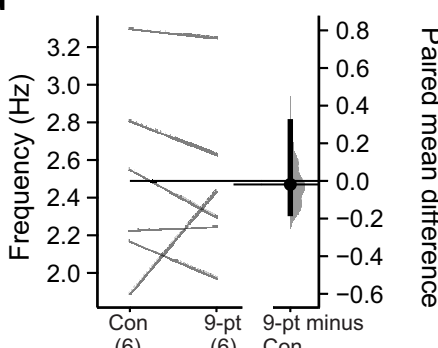

(6) (6) Con

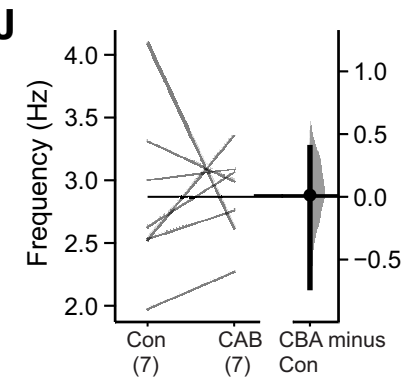

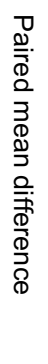

Figure 6. TRPM4 contributes to subthreshold oscillations in SCN neurons. $\boldsymbol{A}$, Action potentials and subthreshold oscillations recorded in the same neuron at $-50 \mathrm{mV}$ (by DC current injection). $\boldsymbol{B}$, Subthreshold oscillations at different membrane potentials in SCN neurons (in TTX, $0.5 \mu \mathrm{M}$ ). $\boldsymbol{C}, \boldsymbol{D}$, Integrated oscillation power $(\boldsymbol{C})$ and frequency $(\boldsymbol{D})$ obtained by FFT for multiple SCN neurons at different membrane potentials; oscillations were clearly observed in $\sim 77 \%$ of SCN neurons at -40 to $-45 \mathrm{mV}(n=24 / 31$, with integrated power $>1$ ). The mean differences from $-55 \mathrm{mV}$ are presented on the lower plots as bootstrap sampling distributions. Data were assessed statistically by two-sided permutation $t$ test. For power: $p=0.0306$ at $-50 \mathrm{mV}, p=0.0004$ at $-45 \mathrm{mV}, p<0.0001$ at $-40 \mathrm{mV}$. For frequency: $p=0.14$ at $-50 \mathrm{mV}, p=0.0554$ at $-45 \mathrm{mV}, p=0.023$ at $-40 \mathrm{mV}$. E-J, Representative effects on subthreshold oscillations in TTX $(0.5 \mu \mathrm{M} T \mathrm{TX}$, at $-40 \mathrm{mV}$ with DC current injection) of bath application of two TRPM 4 channel blockers, 9-pt $(\boldsymbol{E}, 30 \mu \mathrm{M})$ and $\mathrm{CBA}(\boldsymbol{H}, 50 \mu \mathrm{M})$, with summary for all cells showing paired mean differences as bootstrap sampling distributions for effects on integrated power $(\boldsymbol{F}, \boldsymbol{I})$ and mean frequency $(\boldsymbol{G}, \boldsymbol{J})$ after either 9-pt $(\boldsymbol{F}, \boldsymbol{G})$ or CBA $(\boldsymbol{I}, \boldsymbol{J})$. Paired mean differences for power: $p<0.0001$ for 9 -pt and $p=0.0122$ for CBA; and for frequency: $p=0.904$ for 9 -pt and $p=0.967$ for CBA, all by two-sided permutation $t$ test.

are complex, and involve factors other than the L-type calcium channels and TRPM4-dependent large amplitude oscillations that we examined here. For example, we find that L-type channels account for a relatively small fraction of the total $\mathrm{Cd}^{2+}$-sensitive calcium currents evoked by the waveform in LC neurons, even as they are responsible for nearly all of 9-pt-sensitive current and have been shown to strongly inhibit oscillations (Filosa and Putnam, 2003; Imber and Putnam, 2012; Sanchez-Padilla et al., 2014). Although low 


\section{A}

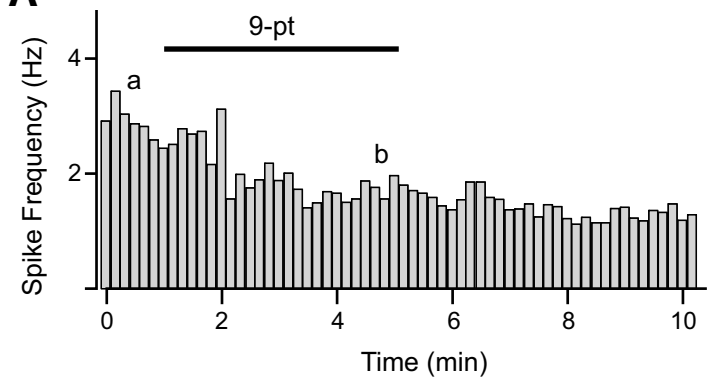

\section{B}

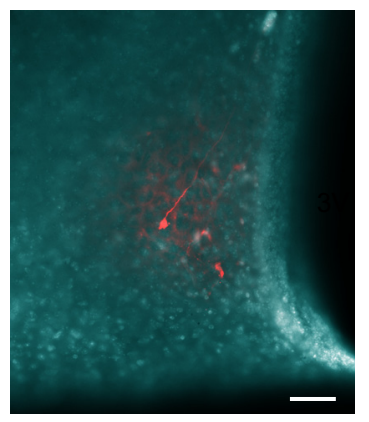

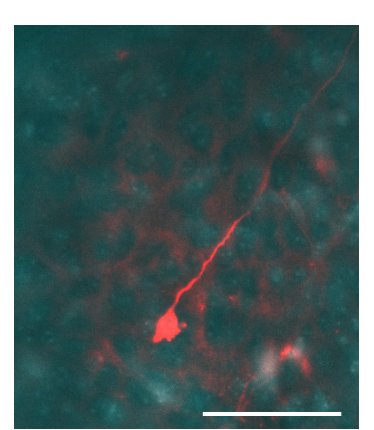

a

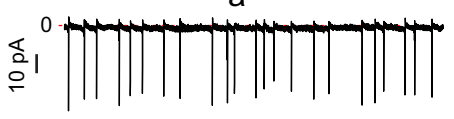

b

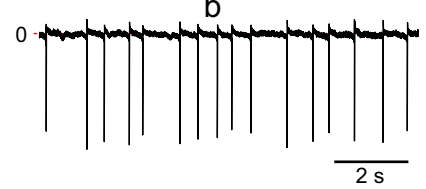

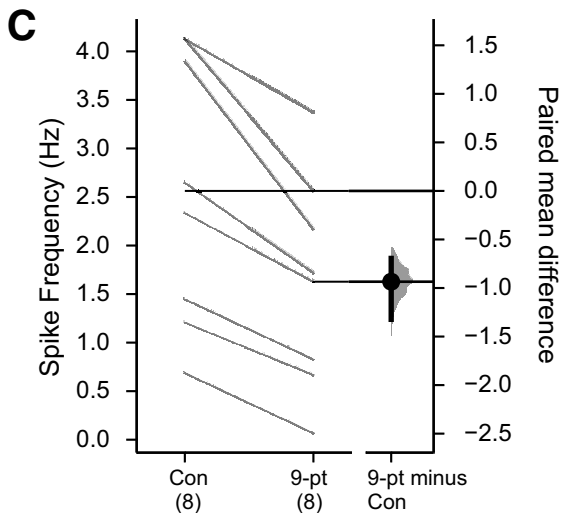

Figure 7. TRPM4 contributes to spontaneous firing in SCN neurons. $\boldsymbol{A}$, Rate histogram (10-s bin averages of instantaneous frequency) of spontaneous action potential firing (no TTX) in an SCN neuron recorded in cell-attached configuration, under control conditions and in the presence of 9-pt $(30 \mu \mathrm{m})$. Example recordings from the indicated time points are provided (right). $\boldsymbol{B}$, Photomicrograph of Lucifer yellow-filled SCN neuron. $\boldsymbol{C}$, Summary data show firing frequency for cells in control and 9-pt and the paired mean difference between control and 9-pt as a bootstrap sampling distribution; $p=0.0096$ by two-sided permutation $t$ test. Scale bars: $50 \mu \mathrm{m}$.

threshold L-type channels play an important role in generating subthreshold oscillations, dihydropyridine L-type channel blockers had only modest or variable effects on baseline action potential firing frequency in LC and SCN neurons (Filosa and Putnam, 2003; Jackson et al., 2004). In SCN neurons, an indirect consequence of L-type channel blockers was reduction of $\mathrm{Ca}^{2+}$-activated $\mathrm{K}^{+}$current, which provided a depolarizing effect to substitute for the loss of calcium current and mitigate effects on firing (Jackson et al., 2004). The modest effects of L-type channel blockers on firing contrast with the relatively consistent and clear effect of TRPM4 blockers on firing frequency that we found here in LC and SCN neurons, and reported earlier in RTN neurons (Li et al., 2021). Interestingly, an important role for NALCN, a "leak" sodium channel, in driving spontaneous firing has also been reported in all three cell groups: LC, SCN, and RTN (Jackson et al., 2004; Sanchez-Padilla et al., 2014; Flourakis et al., 2015; Shi et al., 2016). Thus, we suspect that the large subthreshold oscillations and spontaneous firing in these cells are driven by an interplay between $\mathrm{Ca}^{2+}$ influx carried via low-threshold L-type calcium channels and depolarization mediated by NALCN channels, as integrated and amplified by the combined $\mathrm{Ca}^{2+}$-sensitive and voltage-sensitive TRPM4 cationic channels.

It is also important to note that earlier work characterized additional smaller subthreshold membrane potential fluctuations under both control and TTX conditions at more negative membrane potentials in rat LC neurons; we occasionally observed these small amplitude oscillations but did not examine them in detail. It was suggested that the smaller oscillations may be low amplitude manifestations of the larger "TTX-insensitive spikes" that they also observed at more depolarized potentials (e.g., since both are sensitive to L-type channel blockers; Christie et al., 1989; Filosa and Putnam, 2003; Imber and Putnam, 2012). The smaller oscillations may have been more prevalent in earlier work because of the absence of synaptic blockade and/or recordings performed at more physiological temperatures in a $\mathrm{CO}_{2}-\mathrm{HCO}_{3}{ }^{-}$-based bath solution with elevated $(5 \mathrm{~mm}) \mathrm{K}^{+}$(Christie et al., 1989; Filosa and Putnam, 2003; Imber and Putnam, 2012).

We found subtle differences in how TRPM4 contributes to electroresponsive properties in LC and SCN neurons, perhaps reflecting the complement of supporting ion channels in the different cell groups. For example, TRPM4 blockers reduced the oscillation power in LC and SCN neurons but they lowered the frequency of the residual membrane potential fluctuations only in LC neurons. This suggests a particular role in LC neurons for TRPM4 in the oscillatory mechanism, per se, in addition to its more general amplifying effect on oscillatory power that was observed in the other cell groups. It is also possible that variable contributions from the related $\mathrm{Ca}^{2+}$-activated TRPM5 cation channel (Prawitt et al., 2003), for which there is currently no specific blocker, could account for some of these neuron-specific differences. 


\section{TRPM4 and oscillations in other neurons}

A role for TRPM4 is also possible in other cells where similar oscillations been recorded. For example, SNpc neurons express TRPM4 and the oscillation-dependent tonic firing of SNpc neurons is blocked by 9-pt and flufenamic acid, which also inhibits TRPM4 (Mrejeru et al., 2011). Likewise, TRPM4 mediates a plateau potential and persistent firing in reticular thalamic neurons following burst firing or membrane depolarization that contributes to the slow, $1-\mathrm{Hz}$, oscillations observed in thalamocortical circuits (O'Malley et al., 2020). However, the oscillatory phenomenon (network vs intrinsic), the source for calcium to enable TRPM4 activity (T-type vs L-type) and the ensuing cellular membrane potential property (plateau potential vs subthreshold oscillation) are all very different in this context. Interestingly, we also found that Trpm4 is prominently expressed in MeV neurons, immediately adjacent to LC, and those cells also exhibit prominent subthreshold resonance and oscillations (Wu et al., 2001; Tanaka et al., 2003; Tsuruyama et al., 2013). It should be noted, however, that the characteristics of those $\mathrm{MeV}$ membrane phenomena are different from the oscillations in RTN, LC and SCN neurons, i.e., MeV neurons display $\sim 10-\mathrm{Hz}$ oscillations at hyperpolarized potentials that require $\mathrm{HCN}$ current and $\sim 90-\mathrm{Hz}$ oscillations at depolarized potentials that require TTX-sensitive persistent $\mathrm{Na}_{V}$ current, but not calcium current (Wu et al., 2001; Tanaka et al., 2003; Tsuruyama et al., 2013). Nonetheless, these distinct properties do not preclude a supporting role for TRPM4 current in MeV oscillations, a possibility that has not been tested.

\section{Limitations and caveats}

Some limitations should be acknowledged. First, the experiments were performed on neurons in slices, where voltage-clamp conditions are not ideal; this can be especially of concern in cells with extensive processes or electrotonic coupling, as has been described for neonatal/ adult LC and SCN neurons (Christie et al., 1989; Jiang et al., 1997). That said, such "space clamp" concerns are less acute when analyzing electroresponsive properties with relatively slow kinetics, as is the case with the oscillations we examined. Second, we used two channel blockers, 9-pt and CBA, to identify TRPM4 contributions to subthreshold oscillations in LC and SCN neurons. Although both blockers are reported to be relatively specific for the channel at the concentrations used $(30 \mu \mathrm{M}$ and $50 \mu \mathrm{m}$; Grand et al., 2008; Guinamard et al., 2014; Burris et al., 2015; Ozhathil et al., 2018), we cannot exclude off-target actions of these compounds. Indeed, in vascular cells, 9-pt was reported to activate a calcium-activated $\mathrm{K}^{+}$current (Garland et al., 2015) and inhibit TMEM16A (Burris et al., 2015). In TRPM4 knock-out mice, 9-pt blocked a muscarinic-dependent sADP in pyramidal neurons of prefrontal cortex, implying an off-target action at the $100 \mu \mathrm{m}$ concentration applied (Lei et al., 2014); by contrast, the effects of 9-pt on cardiac pacing were eliminated in TRPM4 knock-out mice, as expected for selective actions on the channel in this context (Guinamard et al., 2015). We are not aware of any reported nonspecific actions of the newer compound, CBA, in either cell systems or knock-out mice. Thus, these concerns are mitigated by the essentially identical actions obtained with two chemically distinct pharmacological blockers of the channel. Finally, we did not examine these oscillatory phenomena in TRPM4 knock-out mice, and we are also unaware of any reports of phenotypes in TRPM4 knockout mice that can be attributed to altered function in LC and SCN neurons; some may yet be discovered.

In conclusion, together with recent work from RTN respiratory chemoreceptor neurons (Li et al., 2021), the current results from LC and SCN neurons support a general role for TRPM4 channels in providing a calcium-dependent and voltage-dependent cationic current to help drive the large subthreshold oscillations that support spontaneous firing in these cells.

\section{References}

Abrahamson EE, Moore RY (2001) Suprachiasmatic nucleus in the mouse: retinal innervation, intrinsic organization and efferent projections. Brain Res 916:172-191.

Bean BP (2007) The action potential in mammalian central neurons. Nat Rev Neurosci 8:451-465.

Bernard C (2019) Changing the way we report, interpret, and discuss our results to rebuild trust in our research. eNeuro 6: ENEURO.0259-19.2019.

Burris SK, Wang Q, Bulley S, Neeb ZP, Jaggar JH (2015) 9Phenanthrol inhibits recombinant and arterial myocyte TMEM16A channels. Br J Pharmacol 172:2459-2468.

Calin-Jageman RJ, Cumming G (2019) Estimation for better inference in neuroscience. eNeuro 6:ENEURO.0205-19.2019.

Card JP, Brecha N, Karten HJ, Moore RY (1981) Immunocytochemical localization of vasoactive intestinal polypeptide-containing cells and processes in the suprachiasmatic nucleus of the rat: light and electron microscopic analysis. J Neurosci 1:1289-1303.

Chan CS, Guzman JN, Ilijic E, Mercer JN, Rick C, Tkatch T, Meredith GE, Surmeier DJ (2007) Rejuvenation' protects neurons in mouse models of Parkinson's disease. Nature 447:1081-1086.

Christie MJ, Williams JT, North RA (1989) Electrical coupling synchronizes subthreshold activity in locus coeruleus neurons in vitro from neonatal rats. J Neurosci 9:3584-3589.

Cui N, Zhang X, Tadepalli JS, Yu L, Gai H, Petit J, Pamulapati RT, Jin $X$, Jiang C (2011) Involvement of TRP channels in the $\mathrm{CO}_{2}$ chemosensitivity of locus coeruleus neurons. J Neurophysiol 105:27912801.

Filosa JA, Putnam RW (2003) Multiple targets of chemosensitive signaling in locus coeruleus neurons: role of $\mathrm{K}^{+}$and $\mathrm{Ca} 2+$ channels. Am J Physiol Cell Physiol 284:C145-C155.

Flourakis M, Kula-Eversole E, Hutchison AL, Han TH, Aranda K, Moose DL, White KP, Dinner AR, Lear BC, Ren D, Diekman CO, Raman IM, Allada R (2015) A conserved bicycle model for circadian clock control of membrane excitability. Cell 162:836-848.

Garland CJ, Smirnov SV, Bagher P, Lim CS, Huang CY, Mitchell R, Stanley C, Pinkney A, Dora KA (2015) TRPM4 inhibitor 9-phenanthrol activates endothelial cell intermediate conductance calciumactivated potassium channels in rat isolated mesenteric artery. $\mathrm{Br}$ J Pharmacol 172:1114-1123.

Grand T, Demion M, Norez C, Mettey Y, Launay P, Becq F, Bois P, Guinamard R (2008) 9-phenanthrol inhibits human TRPM4 but not TRPM5 cationic channels. Br J Pharmacol 153:1697-1705.

Guinamard R, Hof T, Del Negro CA (2014) The TRPM4 channel inhibitor 9-phenanthrol. Br J Pharmacol 171:1600-1613.

Guinamard R, Bouvagnet P, Hof T, Liu H, Simard C, Sallé L (2015) TRPM4 in cardiac electrical activity. Cardiovasc Res 108:21-30.

Häusser M, Raman IM, Otis T, Smith SL, Nelson A, du Lac S, Loewenstein Y, Mahon S, Pennartz C, Cohen I, Yarom Y (2004) 
The beat goes on: spontaneous firing in mammalian neuronal microcircuits. J Neurosci 24:9215-9219.

Imber AN, Putnam RW (2012) Postnatal development and activation of L-type $\mathrm{Ca} 2+$ currents in locus ceruleus neurons: implications for a role for $\mathrm{Ca} 2+$ in central chemosensitivity. J Appl Physiol (1985) 112:1715-1726.

Jackson AC, Yao GL, Bean BP (2004) Mechanism of spontaneous firing in dorsomedial suprachiasmatic nucleus neurons. J Neurosci 24:7985-7998.

Jiang ZG, Yang YQ, Allen CN (1997) Tracer and electrical coupling of rat suprachiasmatic nucleus neurons. Neuroscience 77:10591066.

Lazarenko RM, Milner TA, Depuy SD, Stornetta RL, West GH, Kievits JA, Bayliss DA, Guyenet PG (2009) Acid sensitivity and ultrastructure of the retrotrapezoid nucleus in Phox2b-EGFP transgenic mice. J Comp Neurol 517:69-86.

Lei YT, Thuault SJ, Launay P, Margolskee RF, Kandel ER, Siegelbaum SA (2014) Differential contribution of TRPM4 and TRPM5 nonselective cation channels to the slow afterdepolarization in mouse prefrontal cortex neurons. Front Cell Neurosci 8:267.

Li KY, Putnam RW (2013) Transient outwardly rectifying A currents are involved in the firing rate response to altered $\mathrm{CO}_{2}$ in chemosensitive locus coeruleus neurons from neonatal rats. Am J Physiol Regul Integr Comp Physiol 305:R780-R792.

Li K, Abbott SBG, Shi Y, Eggan P, Gonye EC, Bayliss DA (2021) TRPM4 mediates a subthreshold membrane potential oscillation in respiratory chemoreceptor neurons that drives pacemaker firing and breathing. Cell Rep 34:108714.

Mrejeru A, Wei A, Ramirez JM (2011) Calcium-activated non-selective cation currents are involved in generation of tonic and bursting activity in dopamine neurons of the substantia nigra pars compacta. J Physiol 589:2497-2514.

Mulvey B, Bhatti DL, Gyawali S, Lake AM, Kriaucionis S, Ford CP, Bruchas MR, Heintz N, Dougherty JD (2018) Molecular and functional sex differences of noradrenergic neurons in the mouse locus coeruleus. Cell Rep 23:2225-2235.

Nedergaard S, Greenfield SA (1992) Sub-populations of pars compacta neurons in the substantia nigra: the significance of qualitatively and quantitatively distinct conductances. Neuroscience 48:423-437.

Nedergaard S, Flatman JA, Engberg I (1993) Nifedipine- and omegaconotoxin-sensitive $\mathrm{Ca}^{2+}$ conductances in guinea-pig substantia nigra pars compacta neurones. J Physiol 466:727-747.

O'Malley JJ, Seibt F, Chin J, Beierlein M (2020) TRPM4 conductances in thalamic reticular nucleus neurons generate persistent firing during slow oscillations. J Neurosci 40:4813-4823.

Ozhathil LC, Delalande C, Bianchi B, Nemeth G, Kappel S, Thomet U, Ross-Kaschitza D, Simonin C, Rubin M, Gertsch J, Lochner M, Peinelt C, Reymond JL, Abriel H (2018) Identification of potent and selective small molecule inhibitors of the cation channel TRPM4. Br J Pharmacol 175:2504-2519.

Paxinos G, Franklin KBJ (2001) The mouse brain in stereotaxic coordinates. San Diego: Academic Press.

Pennartz CM, de Jeu MT, Bos NP, Schaap J, Geurtsen AM (2002) Diurnal modulation of pacemaker potentials and calcium current in the mammalian circadian clock. Nature 416:286-290.

Perkins KL (2006) Cell-attached voltage-clamp and current-clamp recording and stimulation techniques in brain slices. J Neurosci Methods 154:1-18.

Prawitt D, Monteilh-Zoller MK, Brixel L, Spangenberg C, Zabel B, Fleig A, Penner R (2003) TRPM5 is a transient $\mathrm{Ca}^{2+}$-activated cation channel responding to rapid changes in $[\mathrm{Ca} 2+]$ i. Proc Natl Acad Sci USA 100:15166-15171.

Puopolo M, Raviola E, Bean BP (2007) Roles of subthreshold calcium current and sodium current in spontaneous firing of mouse midbrain dopamine neurons. J Neurosci 27:645-656.

Sanchez-Padilla J, Guzman JN, Ilijic E, Kondapalli J, Galtieri DJ, Yang B, Schieber S, Oertel W, Wokosin D, Schumacker PT, Surmeier DJ (2014) Mitochondrial oxidant stress in locus coeruleus is regulated by activity and nitric oxide synthase. Nat Neurosci 17:832-840.

Shi Y, Abe C, Holloway BB, Shu S, Kumar NN, Weaver JL, Sen J, Perez-Reyes E, Stornetta RL, Guyenet PG, Bayliss DA (2016) NALCN is a "leak" sodium channel that regulates excitability of brainstem chemosensory neurons and breathing. J Neurosci 36:8174-8187.

Tanaka S, Wu N, Hsaio CF, Turman J Jr, Chandler SH (2003) Development of inward rectification and control of membrane excitability in mesencephalic $V$ neurons. J Neurophysiol 89:12881298.

Todd WD, Venner A, Anaclet C, Broadhurst RY, De Luca R, Bandaru SS, Issokson L, Hablitz LM, Cravetchi O, Arrigoni E, Campbell JN, Allen CN, Olson DP, Fuller PM (2020) Suprachiasmatic VIP neurons are required for normal circadian rhythmicity and comprised of molecularly distinct subpopulations. Nat Commun 11:4410.

Tsuruyama K, Hsiao CF, Chandler SH (2013) Participation of a persistent sodium current and calcium-activated nonspecific cationic current to burst generation in trigeminal principal sensory neurons. J Neurophysiol 110:1903-1914.

Wen S, Ma D, Zhao M, Xie L, Wu Q, Gou L, Zhu C, Fan Y, Wang H, Yan J (2020) Spatiotemporal single-cell analysis of gene expression in the mouse suprachiasmatic nucleus. Nat Neurosci 23:456467.

Wu N, Hsiao CF, Chandler SH (2001) Membrane resonance and subthreshold membrane oscillations in mesencephalic $\mathrm{V}$ neurons: participants in burst generation. J Neurosci 21:3729-3739.

Yung WH, Häusser MA, Jack JJ (1991) Electrophysiology of dopaminergic and non-dopaminergic neurones of the guinea-pig substantia nigra pars compacta in vitro. J Physiol 436:643-667. 